\title{
Strategy for Mitigating DKA Risk in Patients with Type 1 Diabetes on Adjunctive Treatment with SGLT Inhibitors: A STICH Protocol
}

\author{
Satish K. Garg, MD, ${ }^{1,2}$ Anne L. Peters, $M D_{1}^{3}$ John B. Buse, $M D_{1}^{4}$ and Thomas Danne, MD
}

D IABETIC KETOACIDOSIS (DKA) is a serious complication of diabetes that occurs primarily in type 1 diabetes (T1D), although it may also affect patients with other forms of insulin-dependent diabetes and may occur in new-onset type 2 diabetes. Insulin deficiency is associated with an increase in glucagon and excessive lipolysis with increased oxidation of fatty acids to ketone bodies in the liver and ketonemia. Ketosis may advance to metabolic acidosis. For DKA to be diagnosed, both ketosis and acidosis must be present. ${ }^{1-3}$ If not recognized and/or treated early, it can become serious and life-threatening; 168,000 patients were admitted to U.S. hospitals for DKA in 2014. ${ }^{4}$

Recent data from the T1D Exchange, a national registry including $>32,000$ U.S. children, adolescents, and adults with T1D, shed some light on the background risks of DKA. In a 2015 report, $<5 \%$ of participants reported experiencing DKA, which occurred most often in children (3\%-4\%), adolescents $13-17$ years of age (4\%), and young adults $18-25$ years $(5 \%)$, but in $<2 \%$ of older patients. DKA rates generally increased with inadequate glucose control, occurring most often in patients with A1C $\geq 9.0 \%$. Overall, $2.3 \%$ of insulin pump users and $4.3 \%$ of those using multiple daily insulin injections (MDI) reported DKA. ${ }^{5}$ The T1D Exchange 5-year follow-up data (2018) are consistent with these findings and highlight the higher prevalence with increasing A1C values (presented at T1D Exchange Annual meeting, March 2018, Tampa Bay, Florida).

That DKA occurs more frequently among patients using MDI than pumps contrasts with the general consensus that insulin pump use increases the risk of DKA. ${ }^{6}$ It is possible that patients and physicians are reluctant to choose pump therapy in a patient who is nonadherent to intensive insulin therapy. DKA usually occurs due to deliberate or accidental insulin omission or concurrent infections and other physiologic stressors (e.g., pancreatitis, trauma, surgery). Among patients using pumps, the most frequently reported causes of DKA include missed insulin doses and pump malfunctions, including insulin delivery interruptions caused by problems with the infusion set, subcutaneous catheter, or disconnections. ${ }^{6-8}$ These problems were common in the first 5-10 years after insulin pumps were introduced and led many patients and providers to discontinue pump therapy. This prompted technological advances such as hyperglycemic alerts and alarms and improved catheters, as well as patient/ provider education and training, which have significantly reduced DKA risk to the levels seen today. Among MDI users, newer, longer acting basal insulin analogues with a longer half-life and duration of action (glargine U100, glargine U300, and degludec U100 or U200) may reduce the risk of DKA by providing coverage for periods where bolus or correction doses might be missed or insufficient. ${ }^{9-11}$

Recent data from studies of selective sodium glucose cotransporter-2 (SGLT2) inhibitors and a dual SGLT1 and SGLT2 inhibitor as adjuncts to insulin in T1D demonstrate a higher incidence of DKA with these agents than with placebo in some, but not all, studies. ${ }^{12-19}$ SGLT inhibition increases the risk of DKA, even as it reduces A1C, glycemic variability, overall insulin dose, and body weight without increasing severe or total hypoglycemia. ${ }^{14-18,20}$ Based on the case reports of DKA in patients with T1D treated with SGLT2 inhibitors, it is clear that this treatment is being used in practice, although at a low rate (presented at T1D Exchange Annual meeting, March 2018, Tampa Bay, Florida). As diabetes therapeutic paradigms evolve, clinicians should be prepared to educate patients and clinic staff about DKA risks, how best to prevent ketosis from progressing to DKA, and early treatment of DKA.

Due to their insulin-independent mechanism of action, SGLT2 or dual SGLT1 and 2 inhibitors lower total daily insulin dose requirements, which may be expected to increase ketosis. If ketosis is not treated, it may progress to DKA, especially in the setting of insulin resistance associated with

\footnotetext{
${ }^{1}$ Department of Medicine and Pediatrics, School of Medicine, University of Colorado Denver, Aurora, Colorado.

${ }^{2}$ Barbara Davis Center for Diabetes, Aurora, Colorado.

${ }^{3}$ Keck School of Medicine of the University of Southern California, Los Angeles, California.

${ }^{4}$ University of North Carolina School of Medicine, Chapel Hill, North Carolina.

${ }^{5}$ Children's and Youth Hospital Auf der Bult, Hannover Medical School, Hannover, Germany.
} 
illness or infection. SGLT2 inhibitor-mediated increases in sodium reabsorption in renal tubules may interfere with renal clearance of ketone bodies, further increasing circulating ketones. Volume depletion from SGLT2 inhibition may also be a factor, as DKA typically is associated with dehydration, and rehydration is an important component of DKA treatment. ${ }^{1,2}$ Meanwhile, glucagon levels rise in response to SGLT2 inhibition, possibly to compensate for increased urinary excretion of glucose. ${ }^{21,22}$ SGLT2 inhibition also augments preproglucagon gene expression by acting directly on SGLT2 receptors in pancreatic alpha cells. ${ }^{23}$ The resulting increased glucagon levels promote additional ketogenesis in the liver. ${ }^{9}$ DKA in the setting of SGLT inhibition may be associated with euglycemia (due to significant glycosuria with SGLT2 inhibition in the kidneys), complicating the diagnosis of DKA. Without the threshold of marked hyperglycemia, DKA diagnosis may be delayed or missed by patients and clinicians.

Phase 3 clinical trial programs were recently completed for the selective SGLT2 inhibitors dapagliflozin (Efficacy and Safety of Dapagliflozin in Patients with Inadequately Controlled T1D [DEPICT] 1 and 2) and empagliflozin (Empagliflozin as Adjunctive to Insulin in Patients with T1D Mellitus [EASE] 2 and 3), which are approved for type 2 diabetes, and the investigational dual SGLT1 and 2 inhibitor sotagliflozin (inTandem1, 2, and 3). ${ }^{14-18,24}$ The increased risk of DKA with SGLT2 inhibition may not necessarily preclude the use of these agents in combination with insulin therapy, as they have demonstrated significant reductions in glucose, weight, insulin dose, glycemic variability, and blood pressure, as well as a decreased incidence of hypoglycemia in some studies. ${ }^{14-18}$ While we await regulatory decisions, a reexamination of DKA management strategies is worthwhile for the diabetes community. Just as improved clinician and patient education helped reduce the rate of DKA among insulin pump users, a greater understanding of the prevention, early detection, and prompt treatment of DKA may reduce the likelihood of the condition arising or becoming serious, whether patients are using SGLT inhibitor therapy.

Prevention of ketosis is, ideally, the first step. Patients should avoid excess alcohol and very-low-carbohydrate or ketogenic diets (especially if taking an SGLT inhibitor). Those who participate in extreme athletic activities (e.g., marathon running) should do so only in close consultation with their healthcare team, and ketones should be closely monitored during metabolically stressful events, including extreme sports and surgical procedures. SGLT inhibitor therapy should be temporarily discontinued $24 \mathrm{~h}$ in advance of any planned activity that might precipitate DKA-mainly situations that result in decreased insulin doses such as surgery, fasting, reduced carbohydrate intake, or prolonged activity. ${ }^{25}$ SGLT inhibitors should also be withheld for illness and dehydration - any sign or symptoms of physical illness should prompt testing for ketones. When initiating and maintaining SGLT therapy in T1D, clinicians should carefully monitor insulin dose reductions, especially basal insulin decreases (pump or MDI). There are differences in insulin dose reductions with different SGLT inhibitors; for example, patients on selective SGLT2 inhibitors such as dapagliflozin or empagliflozin may have larger reductions of basal insulin than bolus insulin. ${ }^{17,26}$ In contrast, the dual SGLT1 and 2 inhibitor sotagliflozin has been associated with larger reductions of bolus insulin than basal insulin, because SGLT1 inhibition in the proximal gastrointestinal tract leads to a blunting and delay of postprandial hyperglycemia and a lower requirement for bolus insulin. ${ }^{14-16}$

Early recognition of impending DKA is of paramount importance. Usual symptoms include nausea, vomiting, and dehydration associated with excessive thirst and increased urination, fatigue, loss of appetite, malaise, weakness, and rapid breathing or shortness of breath. Routine urine or blood ketone monitoring should be considered, especially during metabolically stressful situations. Blood ketone levels are more accurate for assessing degree of ketonemia than urine ketones. ${ }^{27}$ Renal reabsorption of ketones may lead to lower urinary ketone measurements for a given level of ketosis. ${ }^{25}$ Insulin pump users should check for pump failure or catheter inclusions or kinking and verify that insulin is being administered. When ketosis is present, prompt treatment can reverse ketosis and prevent progression to DKA. Patients should consume at least 30-60 grams of carbohydrates and drink $200-500 \mathrm{~mL}$ of fluid. A correction dose of insulin (1.5 times the usual dose) should be taken at the same time as the carbohydrates and fluids, and additional correction insulin and carbohydrates should be taken every $1-2 \mathrm{~h}$ in the setting of SGLT-associated ketonemia regardless of whether their blood glucose is in the euglycemic range. Patients should call their clinician and recheck ketones every $2-4 \mathrm{~h}$. Finally, the patient should seek medical attention if any of these steps cannot be followed, particularly if the patient is unable to keep down fluids or if ketonemia does not resolve within 4-6 h. Emergency treatment should be sought if symptoms of DKA occur, including abdominal pain, nausea, vomiting, fatigue, malaise, and/or dyspnea.

TABle 1. STICH Protocol Involving Administration OF INSUlin, CARBOHYDRATES, and Fluids Once Ketosis Has Been Confirmed IN The SetTing of SGLT Inhibitor Use IN Combination With Insulin for the Treatment of Type 1 Diabetes

\begin{tabular}{ll}
\hline Step 1 & $\begin{array}{c}\text { Verify ketosis by early symptoms as } \\
\text { described above } \\
\text { Identify conditions that might be } \\
\text { causing ketosis }\end{array}$ \\
& Test for ketones \\
& STICH protocol \\
Step 2 & + \\
& Inject bolus insulin \\
& + \\
& Consume 30 g carbohydrates \\
& + \\
& Hydrate \\
Step 3 & Recheck ketones every $3-4 \mathrm{~h}$ \\
Step 4 & Seek emergency medical care if ketosis \\
& does not resolve or if symptoms of \\
& DKA appear, including abdominal pain, \\
& nausea, vomiting, fatigue, and/or dyspnea
\end{tabular}

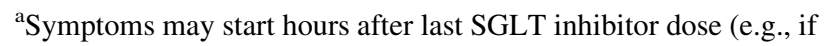
SGLT inhibitor is taken in the morning, symptoms may begin in the evening).

${ }^{\mathrm{b}}$ Patients should not take the SGLT inhibitor after ketosis is detected and not take another dose until ketones have resolved.

DKA, diabetic ketoacidosis; SGLT, sodium glucose cotransporter. 
These steps are summarized in Table 1 , outlining the STICH protocol, a proposed early treatment regimen for patients to begin when they detect high ketone levels. Steps include stopping SGLT treatment for a few days, insulin administration, carbohydrate consumption, and adequate hydration with a suitable drink (water or noncaloric athletic drink with balanced electrolytes).

DKA associated with SGLT2 inhibitors may present with signs and symptoms different from those typically associated with DKA. ${ }^{1-3,25}$ Recent diagnostic criteria can still be used if the patient does not meet a glucose threshold for DKA diagnosis, for example: ${ }^{3}$

- Ketonemia $>3.0 \mathrm{mmol} / \mathrm{L}$ or significant ketonuria (more than $2+$ on standard urine sticks)

- Blood glucose $>200 \mathrm{mg} / \mathrm{dL}$ or known diabetes mellitus

- Bicarbonate $\left(\mathrm{HCO}_{3}{ }^{-}\right)<15.0 \mathrm{mmol} / \mathrm{L}$ and/or venous $\mathrm{pH}$ $<7.3$

It is important to note that blood glucose values may be $<150 \mathrm{mg} / \mathrm{dL}$ (so-called euglycemic DKA), and measurements

A
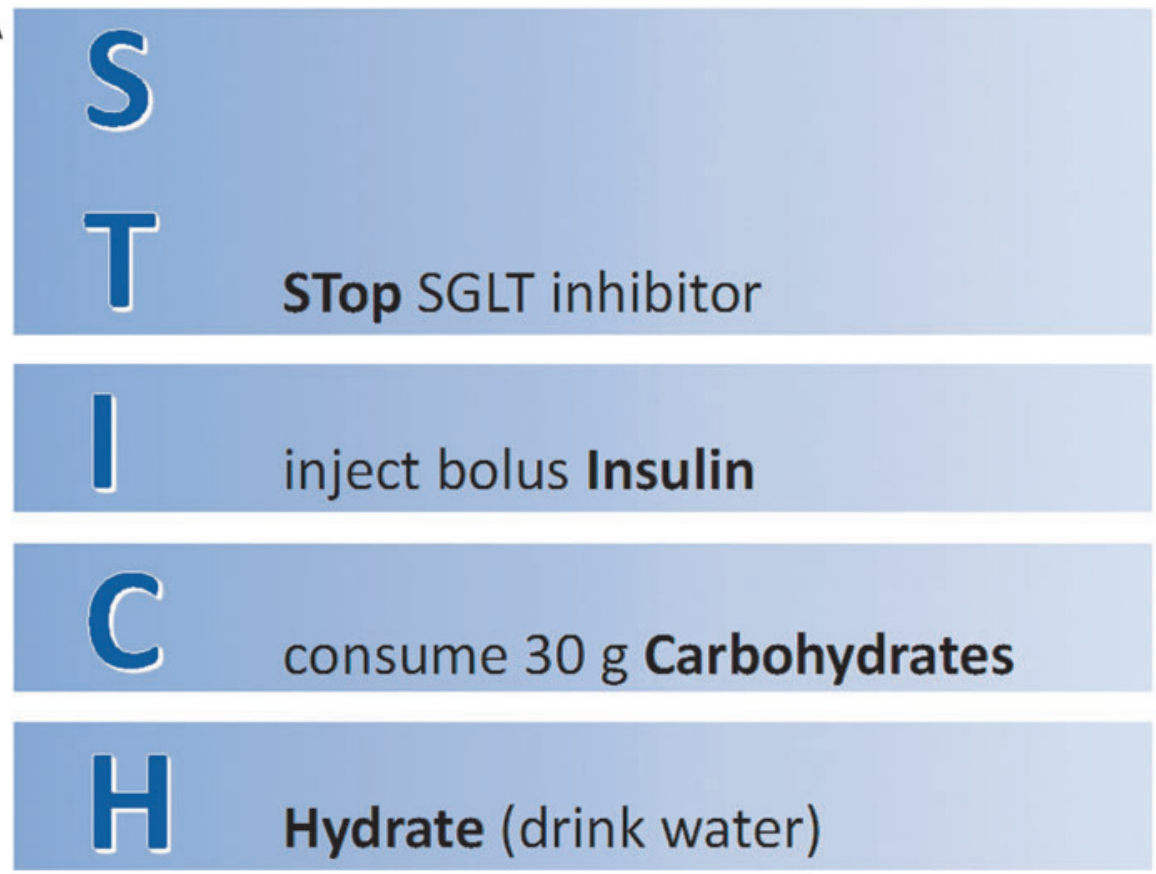

Please carry this card if you are using a SGLT inhibitor with insulin to treat diabetes

B

Diabetic ketoacidosis (DKA) is always preceded by ketosis. It is possible that in patients taking SGLT inhibitors, ketosis or DKA may present with nonspecific or vague symptoms, and normal blood glucose levels, rather than high blood glucose, which is traditionally associated with DKA. Symptoms may include abdominal pain, nausea, vomiting, fatigue, and/or dyspnea. When DKA is suspected, $\beta$-hydroxybutyrate measurements (preferred) or urine ketones should be performed.

To correct ketosis or acidosis, it is vital to ensure that rapid acting insulin is administered frequently regardless of blood sugar levels (even if blood sugar is not elevated). Glucose-containing foods or liquids should be given when this extra insulin is administered.

\section{Patient name:}

If any problems occur, please contact:

Emergency contact:

Phone:

Physician name:

Phone:

FIG. 1. Sample wallet card. (A) Front of card. (B) Back of card. 
of ketonuria and bicarbonate may not accurately reflect patients' metabolic state due to the renal action of SGLT inhibitors. Direct measurement of $\beta$-hydroxybutyrate and $\mathrm{pH}$ is recommended to confirm DKA diagnosis in patients taking SGLT inhibitors. ${ }^{25}$ Patients taking SGLT inhibitors should be advised to carry a wallet card with information about how DKA presentation may differ from standard DKA (Fig. 1). In the emergency department, the same protocol involving carbohydrates, fluids, and insulin should be used to treat DKA.

If off-label use of SGLT2 inhibitors is being considered for patients with T1D, it is important for both clinicians and patients to be aware of conditions that increase the risk of ketosis, and patients must be willing to check ketones using recommended guidelines. Whenever ketone levels are $>3.0 \mathrm{mmol} / \mathrm{L}$, the patient should contact the clinician.

DKA is both preventable and manageable. Mitigating the risks of DKA involves building awareness among clinicians and patients about precipitating factors and how to avoid or minimize their impact. Recent innovations in diabetes treatment, including advances in the design of insulin pumps and use of Continuous Glucose Monitoring (CGM) devices as well as longer acting basal insulin formulations, may reduce DKA risk in patients with diabetes treated with intensive, basal-bolus insulin regimens. Among patients using SGLT inhibitors with insulin (currently off-label in T1D), additional education on how and why SGLT2 inhibition may increase DKA risk - and strategies to appropriately monitor and respond to ketosis - can allow patients with T1D and clinicians to safely use this new adjunctive therapeutic approach if approved by regulatory authorities.

\section{Author Disclosure Statement}

SKG reports receiving grant support and travel support from Sanofi, Lexicon, Novo Nordisk, Mannkind, Roche Diagnostics, Zealand, Senseonics Inc., and Medtronic, and grant support paid to his institution from Eli Lilly, Dexcom, and Johnson \& Johnson.

ALP has participated on advisory boards for Abbott Diabetes Care, Becton Dickinson, Bigfoot, Boehringer Ingelheim, Eli Lilly and Company, Medscape, Merck, Novo Nordisk, Omada Health, and Sanofi; is chair of the type 1 diabetes steering committee at Lexicon; has consulted for Science 37; spoken for and received research supplies from Dexcom; and participated in a speaker's bureau for NovoNordisk.

JBB's contracted consulting fees are paid to the University of North Carolina by Adocia, AstraZeneca, Dance Biopharm, Eli Lilly, MannKind, NovaTarg, Novo Nordisk, Senseonics, and vTv Therapeutics; grant support from Novo Nordisk, Sanofi, and vTv Therapeutics. He is a consultant to Neurimmune AG. He holds stock options in Mellitus Health, PhaseBio and Stability Health. He is supported by a grant from the National Institutes of Health (UL1TR002489).

T.D. has received research support or has consulted for Abbott, AstraZeneca, Bayer, Boehringer, DexCom, Insulet Corp., Eli Lilly, Medtronic, NovoNordisk, Roche, Sanofi, Ypsomed and is a shareholder of DreaMed.

\section{References}

1. Umpierrez G, Korytkowski M: Diabetic emergenciesketoacidosis, hyperglycaemic hyperosmolar state and hypoglycaemia. Nat Rev Endocrinol 2016;12:222-232.
2. Kitabchi AE, Umpierrez GE, Miles JM, Fisher JN: Hyperglycemic crises in adult patients with diabetes. Diabetes Care 2009;32:1335-1343.

3. Dhatariya K, Savage M: The management of diabetic ketoacidosis in adults. 2nd ed. Solihull, West Midlands, UK: Joint British Diabetes Societies Inpatient Care Group, 2013.

4. Centers for Disease Control and Prevention: National Diabetes Statistics Report. Atlanta, GA: Centers for Disease Control and Prevention, U.S. Dept of Health and Human Services, 2017.

5. Miller KM, Foster NC, Beck RW, et al.: Current state of type 1 diabetes treatment in the U.S.: updated data from the T1D Exchange clinic registry. Diabetes Care 2015;38:971-978.

6. Realsen J, Goettle H, Chase HP: Morbidity and mortality of diabetic ketoacidosis with and without insulin pump care. Diabetes Technol Ther 2012;14:1149-1154.

7. Tamborlane WV, Sikes KA: Insulin therapy in children and adolescents. Endocrinol Metab Clin North Am 2012;41: 145-160.

8. Hanas R, Lindgren F, Lindblad B: A 2-yr national population study of pediatric ketoacidosis in Sweden: predisposing conditions and insulin pump use. Pediatr Diabetes 2009; 10:33-37.

9. Taylor SI, Blau JE, Rother KI: SGLT2 inhibitors may predispose to ketoacidosis. J Clin Endocrinol Metab 2015; 100:2849-2852.

10. Barski L, Brandstaetter E, Sagy I, Jotkowitz A: Basal insulin for the management of diabetic ketoacidosis. Eur $\mathbf{J}$ Intern Med 2018;47:14-16.

11. Thalange N, Deeb L, Iotova V, et al.: Insulin degludec in combination with bolus insulin aspart is safe and effective in children and adolescents with type 1 diabetes. Pediatr Diabetes 2015;16:164-176.

12. Peters AL, Buschur EO, Buse JB, et al.: Euglycemic diabetic ketoacidosis: a potential complication of treatment with sodium-glucose cotransporter 2 inhibition. Diabetes Care 2015;38:1687-1693.

13. Fralick M, Schneeweiss S, Patorno E: Risk of diabetic ketoacidosis after initiation of an SGLT2 inhibitor. N Engl J Med 2017;376:2300-2302.

14. Buse JB, Garg SK, Rosenstock J, et al.: Sotagliflozin in combination with optimized insulin therapy in adults with type 1 diabetes: the North American inTandem1 study. Diabetes Care 2018, doi: 10.2337/dc18-0343 [Epub ahead of print]

15. Garg SK, Henry RR, Banks P, et al.: Effects of sotagliflozin added to insulin in patients with type 1 diabetes. $\mathrm{N}$ Engl $\mathbf{J}$ Med 2017;377:2337-2348.

16. Danne T, Cariou B, Banks P, et al.: A1C and hypoglycemia reduction at 24 and 52 weeks with sotagliflozin in combination with insulin in adults with type 1 diabetes: the European inTandem2 study. Diabetes Care 2018, doi: 10.2337/dc18-0342. [Epub ahead of print]

17. Dandona P, Mathieu C, Phillip M, et al.: Efficacy and safety of dapagliflozin in patients with inadequately controlled type 1 diabetes (DEPICT-1): 24 week results from a multicentre, double-blind, phase 3 , randomised controlled trial. Lancet Diabetes Endocrinol 2017;5:864-876.

18. Mathieu C, Dandona P, Gillard P, et al.: Efficacy and safety of dapagliflozin in patients with inadequately controlled type 1 diabetes (the DEPICT-2 Study): 24-week results from a randomized controlled trial. Diabetes Care 2018, doi: 10.2337/dc18-0623. [Epub ahead of print]

19. Garg SK, Strumph P: Effects of sotagliflozin added to insulin in type 1 diabetes. N Engl J Med 2018;378:967968. 
20. Yamada T, Shojima N, Noma H, et al.: Sodium-glucose cotransporter-2 inhibitors as add-on therapy to insulin for type 1 diabetes mellitus: systematic review and meta-analysis of randomized controlled trials. Diabetes Obes Metab 2018; 20:1755-1761.

21. Merovci A, Solis-Herrera C, Daniele G, et al.: Dapagliflozin improves muscle insulin sensitivity but enhances endogenous glucose production. J Clin Invest 2014;124: 509-514.

22. Ferrannini E, Muscelli E, Frascerra S, et al.: Metabolic response to sodium-glucose cotransporter 2 inhibition in type 2 diabetic patients. J Clin Invest 2014;124:499508.

23. Bonner C, Kerr-Conte J, Gmyr V, et al.: Inhibition of the glucose transporter SGLT2 with dapagliflozin in pancreatic alpha cells triggers glucagon secretion. Nat Med 2015;21: 512-517.

24. PRNewswire: Boehringer Ingelheim and Lilly announce positive top-line Phase III data results for empagliflozin as adjunct to insulin in type 1 diabetes. Indianapolis, IN: Eli Lilly and Company, 2018.

25. Handelsman Y, Henry RR, Bloomgarden ZT, et al.: American Association of Clinical Endocrinologists and
American College of Endocrinology position statement on the association of SGLT-2 inhibitors and diabetic ketoacidosis. Endocr Pract 2016;22:753-762.

26. Pieber TR, Famulla S, Eilbracht J, et al.: Empagliflozin as adjunct to insulin in patients with type 1 diabetes: a 4-week, randomized, placebo-controlled trial (EASE-1). Diabetes Obes Metab 2015;17:928-935.

27. Kitabchi AE, Fisher JN, Murphy MB, Rumbak MJ: Diabetic ketoacidosis and the hyperglycemic hyperosmolar nonketotic state. In: Kahn CR, Weir GC, eds. Joslin's Diabetes Mellitus. 13th ed. Philadelphia: Lea \& Febiger, 1994:738-770.

Address correspondence to:

Satish K. Garg, MD

University of Colorado Denver

Barbara Davis Center for Diabetes

1775 Aurora Court, A140

Aurora, CO 80045

Colorado

E-mail: satish.garg@ucdenver.edu 